\title{
PSOÍTE SECUNDÁRIA: RELATO DE CASO EM INDIVÍDUO COM AIDS E REVISÃO DE LITERATURA
}

\author{
SECONDARY PSOAS ABSCESS: CASE REPORT ABOUT A PATIENT \\ WITH AIDS AND REVIEW OF THE LITERATURE
}

Francisco P. Silva1 ${ }^{1}$ Flávio M. Mizoguchi², Renata Y. Saito², José Carlos L. de Souza ${ }^{1}$

1'Docente do Departamento de Clínica Cirúrgica, ${ }^{2}$ Acadêmicos do Curso de Medicina, Universidade Estadual de Londrina - PR. Correspondência: Francisco Pereira Silva. Rua Piaui, 1034 - Centro. CEP 86020-390. Londrina - Paraná - Brasil. (e-mail: uelfpereira@ hotmail.com)

Silva FP, Mizoguchi FM, Saito RY, Souza JCL. Psoíte secundária: Relato de caso em indivíduo com AIDS e revisão de literatura. Medicina (Ribeirão Preto) 2008; 41 (3): 332-8.

RESUMO: Psoíte é uma doença subdiagnosticada, com sintomatologia ambígua, que pode ser responsável pelo retardo no diagnóstico e evoluir de forma grave, constituindo-se em importante ameaça à vida.

O objetivo do trabalho é apresentar caso clínico de psoíte de difícil diagnóstico e discutir os achados deste com a literatura.

Relata-se caso de um indivíduo com 69 anos de idade, casado, internado no serviço de emergências de um Hospital Universitário do Norte do Paraná, que na admissão apresentava sintomas inespecíficos, tais como: febre, emagrecimento e dor abdominal.

O diagnóstico foi realizado utilizando-se dos achados clínicos associados ao exame de ultrassonografia (USG) de abdome total, porém no seguimento também se utilizou da tomografia computadorizada (TC) de abdome.

O tratamento consistiu em drenagem cirúrgica no $8^{\circ}$ dia de internação associada à antibioticoterapia venosa sistêmica. Apesar desta conduta o paciente apresentou abscesso de incisão operatória no 30 을 que foi novamente drenado. Desde então, originou-se uma fístula através desta incisão que se caracterizava por regressões e recidivas. No 59 $\mathrm{PO}$ foi re-internado devido à presença de febre e ao exame físico constatou-se a existência de tumor em quadrante inferior direito e fístula na incisão operatória. Após exame laboratorial foi confirmada a presença de monilíase oral, bacilo da tuberculose na secreção da fístula e sorologia positiva para Vírus da Imunodeficiência Humana (HIV), quando se iniciaram os tratamentos específicos dessas doenças. No seu último retorno ambulatorial, com vinte e sete meses de seguimento, continuava em tratamento para aids, ativo e em excelente estado geral.

Descritores: Abscesso Íliopsoas. aids. HIV. Abscesso do Psoas. Abscesso/Cirurgia.

\section{1- INTRODUÇÃO}

Abscesso de íliopsoas (psoíte) é uma coleção de pus no compartimento do músculo íliopsoas. Tratase de uma condição clínica subdiagnosticada ${ }^{1}$ e que pode apresentar-se com características clínicas vagas, dificultando o diagnóstico. Foi descrito pela primeira vez por Mynter em $1881^{2}$. As causas da psoíte são inúmeras, desde bactérias entéricas Gram negativas a Mycobacterim tuberculosis. Os pacientes imunodeprimidos apresentam o maior risco de desenvolvê-la.

A incidência de psoíte no mundo é desconhecida, contudo parece ter aumentado consideravelmente desde os relatos de Gruenwald em 1992, chegando a 
12 casos $/ \mathrm{ano}^{3}$. Outros pesquisadores concluíram que a doença era subdiagnosticada, porque em um estudo em um único serviço de saúde constatou-se a incidência da doença em 15 pacientes em 3 anos ${ }^{1}$.

O diagnóstico dessa doença antes da tomografia computadorizada era obtido pela necropsia em 70\% dos casos $^{4}$.

A psoíte pode ser classificada em primária e secundária. A primária é provavelmente originária de disseminação hematogênica para o músculo e tem o Staphylococcus aureus o seu principal patógeno (80\% dos casos), além disso, não se conseguiu identificar uma infecção associada em uma estrutura adjacente ao músculo psoas ${ }^{5}$. Dentre as patologias predisponentes como causas primárias temos: o Diabetes Mellitus, o uso de drogas ilícitas, Síndrome da Imunodeficiência Adquirida (aids), insuficiência renal e pacientes imunodeprimidos.

Já na secundária, mais comum em adultos maiores de 50 anos, identifica-se um foco contíguo e infecção como causa do abscesso. ${ }^{6}$ Originando-se em sítios de infecção adjacentes, sendo bactérias do trato gastrintestinal (Escherichia coli e outras enterobactérias) e a tuberculose as causas específicas de abscesso do compartimento íliopsoas. A tuberculose é freqüentemente encontrada em pacientes imunodeprimidos e quando tem como origem a coluna vertebral denomina-se Doença de Pott ${ }^{7}$. A disseminação para a região paravertebral ocorre em cerca de $1-3 \%$ de imunodeprimidos, nesta situação o músculo psoas freqüentemente encontra-se acometido. Entretanto, nem sempre é fácil o estabelecimento do diagnóstico nas fases iniciais do quadro clínico, pois a cultura do material destes sítios costuma ser negativa ${ }^{8}$.

\section{2- RELATO DE CASO}

Paciente masculino, 69 anos de idade, negro, que referia febre diária de $38-39^{\circ} \mathrm{C}$, anorexia, sudorese noturna intensa há 15 dias, sem hemoptise, com emagrecimento de $13 \mathrm{~kg}$ em 2 meses e hipertensão arterial em tratamento, trazendo três exames de escarro negativos nos últimos nove dias.

Antecedente de hipertensão arterial sistêmica em tratamento há 8 meses, prostectomia radical há 3 anos devido a câncer de próstata e apendicectomia há 39 anos.

\section{1- Achados clínico-laboratoriais}

Bom estado geral, peso de $63 \mathrm{~kg}$, estatura de $168 \mathrm{~cm}$ com abdome plano depressível não doloroso a palpação, fígado e baço não palpáveis. No momento da admissão hospitalar o hemograma, cultura de urina, exame de urina e função renal estavam normais, mas a Proteína C Reativa estava alterada.

\section{2- Evolução}

solicitou-se urografia excretora (UGE) que evidenciou compressão e desvio de ureter direito. O exame ultrassonográfico de abdome total realizado no $3^{\circ}$ dia de internação evidenciou a presença de coleção líquida (abscesso) com contornos bem delimitados, com conteúdo heterogêneo medindo $7 \times 3 \mathrm{~cm}$ localizada em fossa ilíaca direita e adenomegalias ao seu redor (Figura 1), com a suspeita de psoíte, foi realizada hemocultura com resultado negativo para aeróbios e anaeróbios. No $6^{\circ}$ dia a tomografia computadorizada (TC) de abdome confirmou a presença de abscesso de psoas (Figura 2) e introduziu-se antibioticoterapia de amplo espectro com ampicilina $1 \mathrm{~g}$ de $6 / 6 \mathrm{~h}$, gentamicina $180 \mathrm{mg} 1 \mathrm{vez}$ ao dia e metronidazol $500 \mathrm{mg}$ de $8 / 8 \mathrm{~h}$ e solicitado consulta ao cirurgião de plantão que no $8^{\circ}$ dia de internação procedeu a drenagem cirúrgica do abscesso retroperitoneal com saída de grande volume de secreção purulenta, com cultura negativa ao exame microbiológico pelo método de cultivo aeróbico qualitativo, e drenagem com o auxílio de sonda Foley até $\mathrm{o} 7^{\circ}$ dia após esta cirurgia, quando novo exame de USG acusou resolução do abscesso e o paciente recebeu alta hospitalar no $27^{\circ}$ dia após esta internação, com medicação sintomática e anti-hipertensivos e cuidados gerais.

Paciente retornou ao Pronto Socorro (PS) no $38^{\circ}$ dia após a $1^{\mathrm{a}}$ internação queixando-se de febre há um dia, dor na incisão operatória e abaulamento local. $\mathrm{Na}$ ocasião, realizou-se drenagem cirúrgica na cicatriz operatória anterior com saída de $15 \mathrm{ml}$ de seroma, deixado um dreno de Penrose no local e introduzido cefalexina, com alta hospitalar no mesmo dia.

No $48^{\circ}$ dia após a $1^{a}$ internação como o paciente referisse febre, foi internado e solicitado nova TC abdômen e parecer do Centro de Controle de Infecção Hospitalar $(\mathrm{CCIH})$ que indicou a introdução de maxcefR e vancomicina. Após 7 dias obteve alta hospitalar com prescrição de ciprofloxacina, pois estava bem e afebril.

No $68^{\circ}$ dia após a $1^{\mathrm{a}}$ internação, foi reinternado com história de febre vespertina há 11 dias, tosse, monilíase oral e fístula na incisão operatória com drenagem espontânea de secreção purulenta. $\mathrm{O}$ exame de USG evidenciou conglomerado de linfonodos ao redor da artéria ilíaca, esplenomegalia e nova coleção 


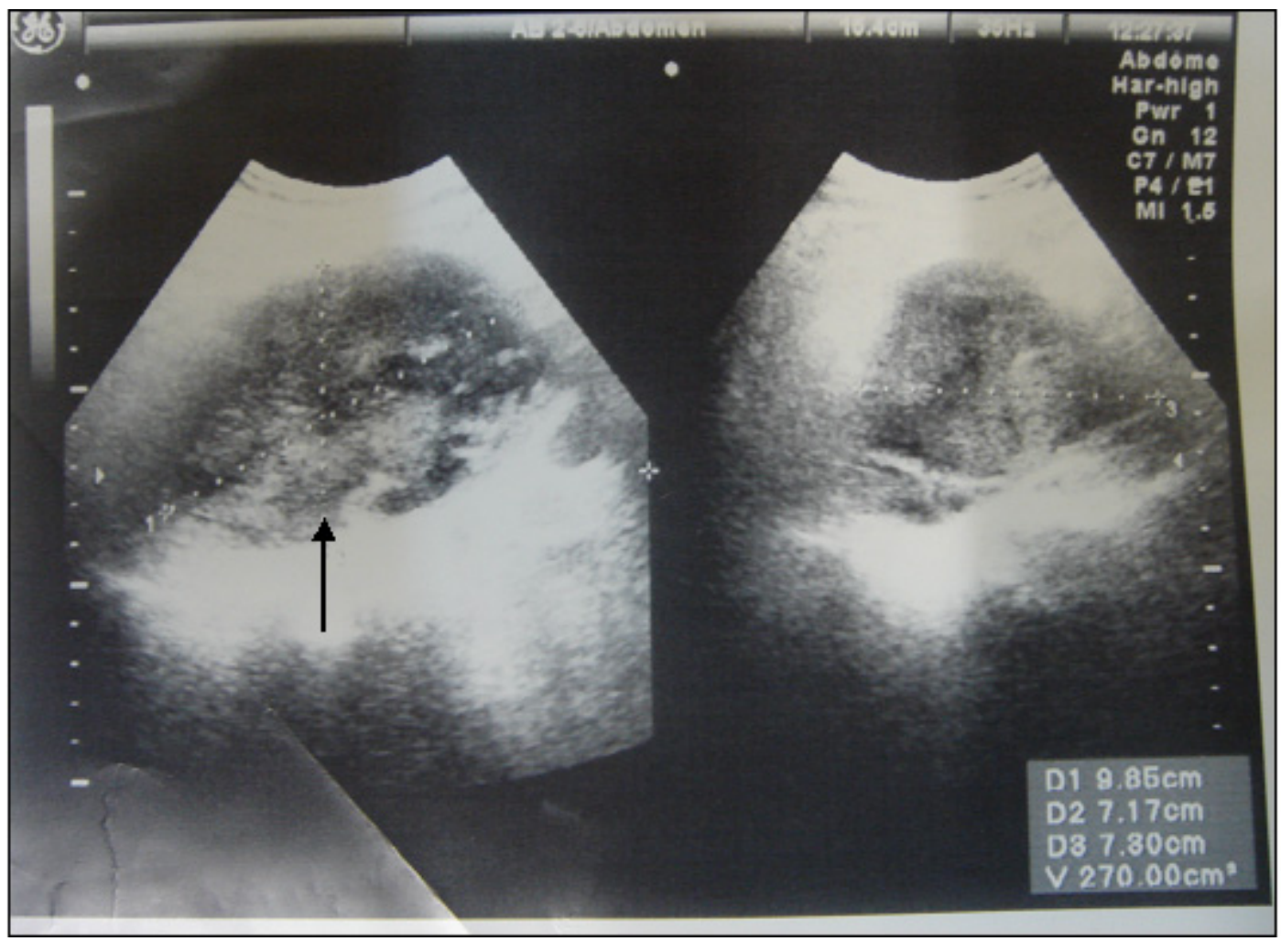

Figura 1: USG de abdômen total evidenciando abscesso com conteúdo heterogêneo em fossa ilíaca direita. A seta indica o abscesso.

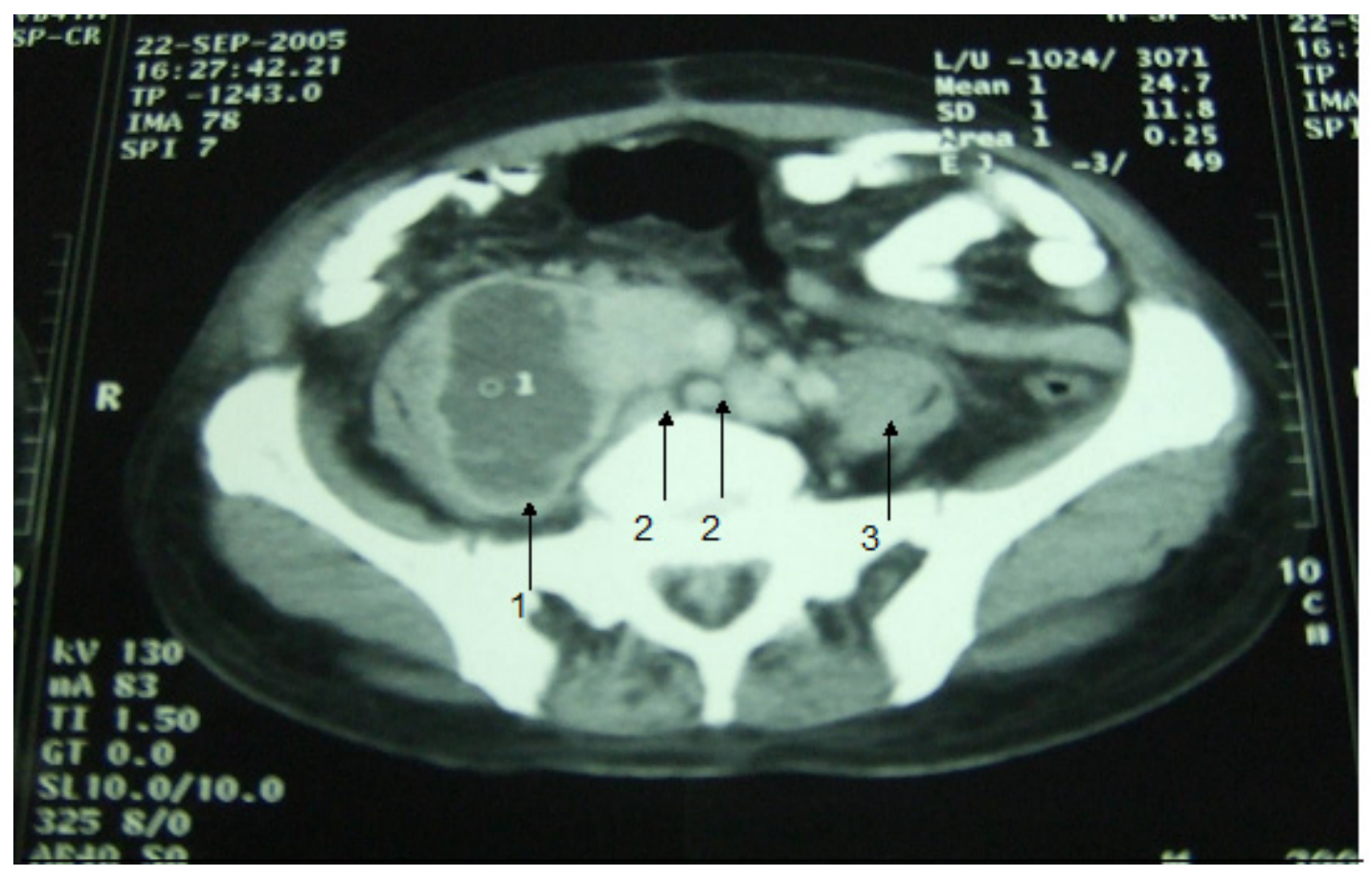

Figura 2: TC de abdômen com presença de abscesso no psoas direito e adenomegalias.

A seta 1 indica o abscesso, as setas 2 as adenomegalias e a seta 3 o psoas esquerdo normal. 
líquida desde incisão da pele até o músculo psoas direito. O exame de cultura e bacterioscopia dessa secreção comprovou Bacilo Álcool Ácido Resistente (BAAR) pela coloração de Ziehl-Neelsin. O exame microbiológico da secreção, pelo método de cultivo aeróbio qualitativo, isolou o microrganismo estafilococo coagulase negativo. Nesta ocasião, foi realizado pesquisa de anticorpos para Vírus da Imunodeficiência Humana (HIV) pelo método de ensaio de imunoabsorção enzimática o qual foi reagente. Um segundo teste pelo método de Micropartículas Enzimáticas também foi reagente para HIV e um terceiro teste confirmatório pelo método de Imunofluorescência Indireta com anticorpos anti-HIV-1 confirmou, enfim, a sorologia positiva para HIV. A pesquisa para bacilo da tuberculose na urina foi negativa. Após estes resultados imediatamente introduziu-se tratamento para tuberculose e para a infecção pelo HIV. Obteve alta hospitalar no $19^{\circ}$ dia de internação.

No $208^{\circ}$ dia após sua última alta hospitalar voltou ao PS com queixa de dor na incisão, a USG evidenciou nova coleção de $5,3 \mathrm{~cm}$ x $5,0 \mathrm{~cm}$ no subcutâneo, sendo efetuada drenagem através da incisão obtendo alta dois dias após, havendo cicatrização da ferida após quatro semanas. No momento, o paciente continua bem e em tratamento para aids e hipertensão.

\section{3- DISCUSSÃO E REVISÃO DE LITERATURA}

O abscesso de psoas é uma doença infecciosa preocupante devido ao potencial de ameaça à vida.

Os sintomas dessa patologia são inespecíficos. A tríade típica de febre, dor em flanco e limitação de movimento do quadril e membros inferiores está presente somente em $30 \%$ dos casos $^{9}$. Outras manifestações clínicas podem ocorrer: dor abdominal, náuseas, vômitos, anorexia, perda de peso e mal estar.

Dentre as situações clínicas associadas com o abscesso do psoas ilíaco tem-se:

1- Gastrintestinal: doença de Crohn, diverticulite, apendicite e o câncer colorretal;

2- Genito-urinário: infecção do trato urinário, câncer, litotripsia extra-corpórea;

3- Músculo-esquelético: osteomielite vertebral, artrite séptica, sacroileíte;

4- Vascular: aneurisma aórtico abdominal infectado e cateterização de vasos femurais;

5- Miscelânea: endocardite, dispositivos de anticoncepção intrauterino, linfandenite supurativa. ${ }^{9}$
Alguns autores têm descrito duas formas clínicas desta doença: uma forma chamada típica na qual a doença se manifesta no paciente com febre, dor lombar ou inguinal; e outra atípica e insidiosa se apresentando com febre e mal estar abdominal ${ }^{10}$. No caso descrito o paciente inicialmente apresentou quadro atípico de psoíte que posteriormente com o aparecimento da dor inguinal evoluiu como forma típica associada a quadro consuptivo e febre persistente.

Pouco pensada, esta doença é uma condição que pode ser extremamente difícil de diagnosticar rapidamente, devido à inespecificidade do quadro. Esta característica leva frequientemente a uma demora no diagnóstico que em média é de 45 dias ${ }^{11}$. No caso em estudo, o paciente foi diagnosticado após treze dias da primeira consulta e após sete dias de internação, por outro lado, a hipótese de infecção pelo HIV foi aventada e confirmada somente após dois meses de acompanhamento médico.

Os exames laboratoriais que auxiliam no diagnóstico do quadro infeccioso, como o hemograma acusando leucocitose (acima de 15900/ $\mathrm{mm}^{3}$ ), a elevação de Velocidade de Hemossedimentação (VHS) (> $90 \mathrm{~mm} / \mathrm{h}$ ) e elevação de uréia( $305 \mathrm{mg} / \mathrm{dl}$ ) não são achados universais ${ }^{12}$, sendo que o aumento desta última sugere etiologia aguda ou sub aguda ${ }^{12}$. No nosso paciente apenas o VHS persistiu aumentado desde sua primeira consulta até seu último retorno ambulatorial.

O exame radiográfico simples de abdômen é um método não invasivo que pode evidenciar a presença de coleções retroperitoneais, borramento da região do músculo psoas e ar em retroperitônio realçando o contorno duodenal e/ou renal. Na psoíte este exame está alterado em aproximadamente $30 \%$ dos casos. Portanto, o valor da radiografia simples para o diagnóstico de abscesso do psoas é limitado ${ }^{13}$. No nosso paciente observaram-se todos os sinais exceto a presença do ar em retroperitônio.

A USG é um exame não invasivo de fácil realização, útil tanto para o diagnóstico, quanto para orientação terapêutica como a punção e/ou drenagem ${ }^{12}$. Neste paciente, os exames ultrassonográficos foram suficientes para apontar o diagnóstico de psoíte.

A TC é um excelente método diagnóstico e tem vantagens em relação ao USG, principalmente quando o abdômen se encontra com distensão de gases na luz entérica. A TC também é utilizada para a orientação das punções e drenagens do abscesso de psoas. No nosso hospital o exame de imagem mais utilizado 
para punções ou drenagens em paciente com suspeita de psoíte é a USG, que é o método de imagem que proporciona diagnóstico em $60 \%$ dos $\operatorname{casos}^{14,15}$; entretanto, em pacientes obesos a dificuldade de diagnosticar pequenos abscessos é maior com este método16 do que na TC .

A TC com contraste é a técnica de imagem de maior valor para o diagnóstico da psoíte com sensibilidade de $100 \%^{13,17,18,19}$. No caso em tela, utilizou-se da TC pela primeira vez no $9^{\circ}$ dia de internação devido à persistência do quadro febril, mas ela não acrescentou novas informações em relação ao USG prévio. A TC é um exame de grande valia também para controle evolutivo desses pacientes na busca de outras coleções residuais ou acometimento de outras estruturas como a coluna vertebral, dessa forma, a TC possibilitou a exclusão de Mal de Pott.

A Doença de Pott é mais comum em pacientes soropositivos para o HIV20, que é detectado em até $60 \%$ dos $\operatorname{casos}^{21}$. A imunossupressão associada a esses indivíduos permite uma disseminação da infecção pelo Mycobacterium tuberculosis e aumento na incidência de tuberculose extrapulmonar ${ }^{22}$. Os exames de eleição para investigação diagnóstica da Doença de Pott são a TC e a ressonância magnética (RNM). A TC é mais acessível, e quando detecta a presença de focos de calcificação dentro do abscesso é patognomônico para tuberculose, além de possibilitar a aspiração guiada via percutânea ${ }^{23}$, pois, permite uma melhor visualização de tecido ósseo em relação à RNM. No nosso paciente o exame radiológico simples de coluna e a TC realizados no $8^{\circ}$ e $9^{\circ}$ dias de internação, respectivamente, excluíram a hipótese de Mal de Pott e o diagnóstico de tuberculose só foi confirmado após dois meses da primeira internação com o auxílio do exame de secreção de fistula da incisão operatória.

Alguns autores acreditam também que a utilização da RNM seja superior a TC devido a sua melhor capacidade discriminativa do tecido mole, a facilidade de possibilitar a visualização da parede do abscesso e as estruturas que o delimitam sem a utilização de contrastes endovenosos ${ }^{24}$.

Preconiza-se para o tratamento da psoíte o uso de antibióticos de amplo espectro associado a drenagem do abscesso do músculo psoas, sendo que a antibioticoterapia deve preceder a cirurgia ${ }^{12}$. Esta drenagem pode ser percutânea guiada por USG ou TC. No nosso Hospital a via cirúrgica é a convencional, sendo que a via mais freqüentemente utilizada é a incisão extraperitoneal da fossa ilíaca comprometida, como se fosse a incisão de Kocher, no ponto de Mc Burney, comumente utilizada para realização de apendicectomia. Esta via cirúrgica deve ser utilizada quando falhar a via percutânea, ou quando o abscesso for multiloculado. Atualmente a TC e a drenagem percutânea devem ser consideradas os procedimentos de eleição para o diagnóstico e o tratamento desta moléstia $^{12}$.

A técnica de drenagem percutânea produz resultados comparáveis ao tratamento cirúrgico convencional $^{8,25-28}$.

O controle da drenagem desta doença deve ser realizado com avaliações clínicas e métodos de diagnóstico por imagem, os mais usados são USG e TC. A drenagem deve continuar até a obliteração da cavidade do abscesso e a evidência da melhora clínica do paciente.

A psoíte primária tem melhor prognóstico com mortalidade em torno de $2,4 \%$, ao passo que a secundária tem taxa de mortalidade de $19 \% .{ }^{24} \mathrm{~A}$ mortalidade em pacientes não tratados alcança a taxa de $100 \%{ }^{15}$.

$\mathrm{Na}$ Ásia e na África mais de 99\% das psoítes são primárias, enquanto que respectivamente na Europa e na América do Norte, $17 \%$ e $61 \%$ são primários15. Esta doença é tão freqüente no jovem quanto no idoso3. A mortalidade em grandes séries variou de $7 \%$ a $20 \%{ }^{13,19}$.

De acordo com vários autores é no lado direito que se diagnostica o maior número de abscesso de psoas, no entanto, não existem explicações claras para este fato $^{12}$.

Autores sugerem antibioticoterapia antes do início da drenagem com cefotaxima 2g/4-6 horas ou cloxacilina $2 \mathrm{~g} / 4-6$ horas por via endovenosa associada a 500mg/6-8 horas de metronidazol por via endovenosa e, às vezes, abscessos menores que $5 \mathrm{~mm}$ poderíamos inicialmente tratar apenas com antibióticos sem drenagem ${ }^{12}$. Outros autores sugerem que se deve começar o tratamento desta patologia com antibióticos de amplo espectro como a clindamicina, penicilina e aminoglicosídeo por até duas semanas após a drenagem do abscesso. ${ }^{5}$ Em trabalho realizado por Navarro e colegas em pacientes com psoíte e aids a terapia utilizada foi cloxacilina intravenosa e gentamicina por quatro a seis semanas. ${ }^{29}$

A dor abdominal e febre é a apresentação clínica de psoíte em pacientes infectados pelo HIV. O 
aumento da contagem de leucócitos é raramente observado. O nível de imunossupressão em pacientes com infecção pelo HIV com psoíte é tipicamente severo. E nesses pacientes bactérias gram-negativas e enterobactérias são raramente isoladas como agentes etiológicos. ${ }^{29}$

A presença de tuberculose é maior em pacientes soropositivos para o HIV do que na população geral, especialmente a tuberculose extra-pulmonar ${ }^{30}$. Além disso, a disseminação óssea da tuberculose também é muito mais comum em soropositivos para o HIV, acometendo até $60 \%$ dos $\operatorname{casos}^{21}$.

\section{4- CONCLUSÃO}

É importante incluir a psoíte no diagnóstico diferencial de pacientes com aids que apresentam febre de origem indeterminada. A Doença de Pott é mais comum em pacientes infectados com o HIV, cuja imunossupressão facilita a disseminação do Mycobacterium tuberculosis e aumento na incidência de tuberculose extrapulmonar, contudo, no caso apresentado não foi diagnosticado Doença de Pott e encontrou-se o bacilo da tuberculose apenas na secreção da fístula 60 dias após a drenagem do abscesso.

Silva FP, Mizoguchi FM, Saito RY, Souza JCL. Secondary psoas abscess: case report about a patient with AIDS and review of the literature. Medicina (Ribeirão Preto) 2008; 41 (3): 332-8.

ABSTRACT: Psoas abscess is a disease which is often misdiagnosed, since it has ambiguous symptoms; this fact may be responsible for delays in diagnostic and consequent evolution to a serious form of the disease, which may be a threat to the patient's life.

The purpose of this work is to present a clinical case of psoas abscess of difficult diagnostic and discuss the findings in this case and in the literature.

The work reports a case involving a 69 year old married male patient, who was interned in the emergency unit at Hospital Universitário do Norte do Paraná. At admission the patient presented symptoms which were not specific, such as fever, loss of weight and abdominal pain.

The diagnostic was carried out by utilizing the clinical findings associated to a complete abdominal ultrasound scan (USS); however, in the follow up phase an additional computerized tomography (CT) of the abdomen was utilized.

Treatment consisted of surgical draining carried out on the 8th day of internment associated to systemic venous antibiotic therapy. In spite of such treatment the patient presented a surgical incision abscess on the 30th day after surgery, which was drained again. Since then, a fistula originated at such incision, which was characterized by regressions and relapses. On the 59th day after surgery, the patient was again interned due to fever, and the physical examination revealed the existence of a tumor in the lower right hip and a fistula on the surgical incision. After laboratory exams, the presence of oral moniliasis was confirmed, as well as tuberculosis bacillus in the secretion of the fistula, and positive serology to Human Immunodeficiency Virus (HIV) and specific treatment for these diseases were started. On his latest return to the ambulatory, after twenty seven months of follow up, the patient continued to be treated for AIDS; nevertheless, he was enjoying excellent health and was active again.

Keywords: Psoas Abscess. Iliopsoas Abscess. AIDS. HIV. Abscess/Surgery.

\section{REFERÊNCIAS}

1 - Garner JP, Meiring PD, Ravi K, Gupta R. Psoas abscess - not as rare as we think? Colorectal dis 2007; 9(3):269-74.

2 - Mynter H. Acute psoitis. Buffalo Med Surg 1881;21:202-10 .

3 - Gruenwal I, Abrahamson J, Cohen O. Psoas abscess: case report and review of the literature. J urol 1992; 147: 1624-28.
4 - Altemeier WA, Alexander JW. Retroperitoneal abscess. Arch surg 1961:83:512-24.

5 - Mallick IH, Thoufeeq MH, Rajendran TP. Ilipsoas abscesses. Postgrad med j 2004;80:459-62.

6 - Franco-Paredes C, Blumberg HM. Psoas muscle abscess caused by Mycobacterium tuberculosis and Staphylococcus aureus: Case report and review. Am j med scien 2001; 321: $415-17$. 
7 - Donovan PJ, Zerhouni EA, Siegelman SS. CT of the psoas compartment of the retroperitoneum. Semin Roentgenol 1981;16: 241-50.

8 - Pómbo F, Martín-Egaña R, Cela A, Díaz JL, Linares-Mondéjar $P$, Freire M. Percutaneous catheter drainage of tuberculous psoas abscesses. Acta Radiol. 1993;34: 366-88.

9 - Chern CH, Hu SC, Kao WF, Tsai J, Yen D, Lee CH. Psoas abscess: making an early diagnosis in the ED. Am j emerg med 1997;15: 83-8.

10 - Pigrau C, Pahissa A. Abscesso de psoas: una enfermedad enigmática? Med clin (Barcelona) 1990; 95: 456-58.

11 - Salvatore A, Pavlovsky M, Maxit M, Wouters L, Valdivia H. El absceso del músculo psoas ilíaco.Medicina (Buenos Aires)1996; 56(2): 126-32.

12 - Penedo S, Espigna B, Campo JF. Absceso de psoas. Descripcion de una serie de 23 casos Enferm infecc microbiol clin 2001; 19: 257-60.

13 - Córdoba J, Pigrau C, Pahissa A, Almirante B, Passer I, Martínez-Vázquez JM. Absceso de psoas: utilidad diagnóstica y terapéutica de la ecografía y de la tomografía computadorizada. Med clin (Barcelona). 1992 ;99: 568-70.

14 - Huang JJ, Ruaan MK, Lan RR, Wang MC. Acute pyogenic iliopsoas abscess in Taiwan: clinical features, diagnosis, treatments and outcome. J infect 2000; 40: 248-55.

15 - Ricci MA, Rose FB, Meyer KK.Pyogenic psoas absces: worldwide variations in etiology. World j surg 1986;10:83443.

16 - Hriack H, Boswell W, Henderson R. TC of inflamatory disease of the psoas muscle. AJR 1980; 134: 767-70.

17 - García Vázquez E, Gutiérrez Guisado J, Díaz Curiel M. Abscesos del psoas: presentación de ocho casos y revisión de la literatura. Rev clin esp. 1995; 195: 289-93.

18 - Jiménez-Mejías ME, Alfaro MJ, Bernardos A, Cuello JA, Cañas E, Reyes MJ. Absceso del psoas: uma entidad no tan infrecuente. Analisis de una serie de 18 casos. Enferm infecc microbiol clin. 1991; 9: 148-53.

19 - Laguna P, Moya M. Absceso del músculo psoas: análisis de 11 casos y revisión de la bibliografía. Enferm infecc microbiol clin. 1998;16:19-24.

20 - Centers for Disease Control and Prevention. Expanded tuberculosis surveillance and tuberculosis morbidity. United States. Morbidity and Mortality WeeKly Report 1994; 43: 361-6.
21 - Moon MS. Tuberculosis of the spine: Controversies and a new challenge. Spine(Hagerstown) 1997; 22(15): 1791-7.

22 - Haas DW. Mycobacterium tuberculosis. In: Douglas M, Bennett's (eds) Principles and Practice of Infections Diseases, 5th edtition, Churchill Livingstone, Chapter 240, 2000.

23 - Griffth JF, Kumta SM, Leung PC, Cheng JCY, Chow LTC, Metreweli C. Imaging of Musculoskeletal Tuberculosis: A New Look at an Old Disease. Clin orthop relat res 2002; 398: 32-9.

24 - Qureshi NH, O'Brien DP, Allcutt DA. Psoas abscess secondary to discitis: a case report of conservative management. $J$ spinal disord 2000: 13: 73-6.

25 - Cantasdemir M, Kara B, Cebi D, Selcuk ND, Numan F. Computed tomography-guided percutaneous catheter drainage of primary and secondary iliopsoas abscesses. Clin radiol. 2003; 58: 811-15.

26 - De Gregorio MA, López S, Fernández JA, Pérez J, Ariño I, Alfonso ER, et al. El drenaje percutáneo como tratamiento de los abscesos del músculo psoas.Cir esp. 1995; 57:56570 .

27 - De Miguel J, Miguélez JL, Mayo J, Collazos J, Martínez E, Marcos A. Absceso de psoas. Consideraciones diagnósticas y terapéuticas en cinco pacientes. Rev clin esp. 1993; 192: 123-6.

28 - Navarro V, Meseguer V, Fernández A, Medrano F, Sáez JA, Puras A. Absceso del músculo psoas. Descripción de una serie de 19 casos. Enferm infecc microbiol clin. 1998;16: 118-22.

29 - Navarro V, López F., González E., Gregori J., Muñoz A. Psoas abscess in patients infected with the human immunodeficiency vírus. Eur J Clin Microbiol Infect Dis. 2004; 23: 661-3.

30 - Kozakis L, Ballachandran T. Bilateral psoas abscess in an HIV-positive patient. Int j STD AIDS 2004; 15: 841-43.

Recebido para publicação em 10/12/2007

Aprovado para publicação em 29/09/2008 